\title{
CUERPO Y TRAGEDIA
}



Daimon. Revista Internacional de Filosofía, Suplemento 5 (2016), 857-866

ISSN: 1130-0507 (papel) y 1989-4651 (electrónico)

http://dx.doi.org/10.6018/daimon/268901

\title{
El cuerpo de Electra: una lectura política de la tragedia
}

\author{
Electra's Body: \\ a Political Reading of the Tragedy
}

CRISTINA BASILI

\begin{abstract}
Resumen: En 1936, la filósofa francesa Simone Weil (1909-1943) escribe un pequeño ensayo sobre la Electra de Sófocles. El texto forma parte de un proyecto más amplio: presentar las obras maestras de la literatura griega a la clase trabajadora. Sin embargo, el breve escrito no representa solamente un admirable ejemplo de divulgación, sino que apunta hacia muchas de las intuiciones políticas de la filósofa. El presente trabajo pretende investigar las conexiones entre la representación de Electra como figura de la opresión y la posición de Weil respecto a la moderna concepción de la política.

Palabras clave: Weil, Electra, Sófocles, Arendt, política, poder.
\end{abstract}

\begin{abstract}
In 1936, French philosopher Simone Weil (1909-1943) writes a short essay on Sophocles' Electra. That was part of a broader project intended to make accessible to the working class the masterpieces of ancient Greek literature. But the composition is not only an admirable example of divulgation; it also represents a key work where most of the Weilian intuitions on power and politics all condense around the suffering body of Electra. The aim of this paper is to show the close connection between the attention to Electra as a figure of oppression and the Weilian critical position towards the modern representation of politics.
\end{abstract}

Keywords: Weil, Electra, Sophocles, Arendt, Politics, Power.

El presente trabajo pretende analizar el breve comentario a la Electra de Sófocles escrito por Simone Weil en 1936 (cf. Weil, 1991a). En el texto, que proporciona una lectura política de la tragedia, el cuerpo de Electra representa tanto el médium a través del cual se manifiesta una radical crítica del poder, cuanto una herramienta de lucha y resistencia. El pequeño escrito revela, gracias a una cuidadosa selección de los pasajes comentados, la significación política del cuerpo de Electra que, en la interpretación de Weil, se transforma en figura o

Fecha de recepción: 10/06/2016. Fecha de aceptación: 12/09/2016.

* Doctora en Humanidades por la Universidad Carlos III de Madrid (cbasili@hum.uc3m.es). Su investigación se centra en la historia del pensamiento político y en la filosofía política moderna y contemporánea. Entre sus publicacione recientes: "Simone Weil, l'ebraismo e la critica della teologia politica", en: O. Ombrosi (ed.), Ebraismo al femminile, Giuntina, Firenze (en curso de publicación); "El límite de política: Hannah Arendt y la polis griega", en: D. Hernández de La Fuente (ed.), De ö $\rho$ o a limes: el concepto de frontera en el mundo antiguo y su recepción, Escolar y Mayo Editores, Madrid (en curso de publicación). 
imagen de la opresión. Sin embargo, hay otra razón más por la que cabe analizar un texto en apariencia marginal dentro de la producción de su autora: una posible línea de investigación que trascendería el pensamiento de la propia Weil. Si se entiende que la tragedia se sirve de un cuerpo de mujer como vehículo de representación de la opresión violenta del poder, se puede afirmar que, desde el punto de vista femenino, el poder nunca ha sido otra cosa que biopoder: poder que ejerce su violencia sobre la vida (cf. Bottici, 2015, 184-186). El ángel de la historia de Weil mira hacia el pasado y, asumiendo como suya la perspectiva de un cuerpo doliente y femenino, trata de rescribir una historia cuyo tiempo no esté marcado por el ritmo del poder, sino por el de su resistencia.

\section{Cuerpo y trabajo}

El pequeño ensayo sobre la Electra, aunque muy sencillo en apariencia, permite arrojar luz sobre una fase de cambios sustanciales en el pensamiento weiliano. La filósofa empieza, en esa época, a delinear de manera cada vez más original su propio recorrido intelectual, influida en buena medida por su asidua lectura de los clásicos griegos. En el texto sobre la tragedia confluyen, en realidad, escondidas detrás de la forma divulgativa, las reflexiones sobre el trabajo obrero que la pensadora había venido desarrollando desde el comienzo de los años 30 (cf. Weil, 1991b).

La cuestión del trabajo es una constante en el pensamiento de Weil (cf. Chenavier, 2001). Intérprete y crítica del pensamiento de Marx, la filósofa considera que la centralidad del trabajo es la gran conquista intelectual de su época (cf. Weil, 2013, 189) y estima que una de las tareas filosófico-políticas fundamentales es su emancipación a partir de la superación de la fractura entre trabajo manual y trabajo intelectual. Para usar sus términos, cabe pensar cuales las condiciones gracias a las cuales el trabajo deje de ser una actividad servil (cf. Weil, 2013, 358-365).

Según ella, el trabajo es la principal herramienta a través de la cual el hombre llega a conocer el mundo: en la manipulación y en la modificación de la realidad llevada a cabo por medio del trabajo está encerrada la posibilidad de su emancipación, junto con el sentido auténtico de la libertad, puesto que la libertad no es lo contrario de la necesidad: más bien, es mediación, conocimiento, comprensión y aceptación de esta. Aquellos que se dedican al trabajo manual tienen, por tanto, que estar en condiciones de tomar conciencia del potencial liberador inscrito en su actividad: «Les travailleurs savent tout; mais hors du travail ils ne savent pas qu'ils ont possédé toute la sagesse» (Weil, 1988, 217). De esto modo, el problema del trabajo y de los trabajadores se configura como el problema político fundamental y, como consecuencia de esta centralidad, la filósofa piensa en una ontología basada en la idea de necesidad en tanto que ley del mundo material, natural y social (cf. Fuster, 2013, 40).

$\mathrm{Si}$, en este punto, se considera el análisis que Hannah Arendt desarolla acerca de las actividades humanas y se utiliza como herramienta crítica para visualizar la posición de Weil, resultará más fácil detectar aquel elemento que une el trabajo al cuerpo y al nivel elemental de la vida biológica. Un ámbito que en el pensamiento de Arendt queda fuera de lo político y que en el de Weil se transforma en el centro de su representación del cuerpo dolido como 
imagen de la opresión ${ }^{1}$, lo que permite hacer visible cómo en Weil el cuerpo incluido en la categorización de la política -y esta es la hipótesis que se quiere desarrollar- funciona como dispositivo crítico de su representación moderna².

Para lo que aquí nos concierne, bastará con recordar brevemente cómo, en la obra The Human Condition -cuyo título, se ha hecho notar, podría evocar justo La condition ouvrière de Weil (cf. Fuster, 2013: 34)- Arendt divide el ámbito de la actividad humana en tres grandes grupos: el trabajo (labour), la obra (work) y la acción (action). El trabajo, "the labour of our body" (Arendt, 1998:79), resulta distinto de la obra, "the work of our hands" (Arendt, 1998:79), en tanto que está relacionado con la esfera de la reproducción, mientras que la obra está relacionada con la esfera de la producción. Lo que caracteriza al trabajo es el hecho de estar relacionado con la dimensión corporal y con sus funciones: con el mantenimiento del nivel elemental, primitivo y biológico de la vida humana y, por tanto, de pertenecer a la dimensión de la necesidad, entendida como opuesta a la dimensión política de la libertad:

The ancients [...] felt it necessary to possess slaves because of the slavish nature of all occupations that served the needs for the maintenance of life. It was precisely on these grounds that the institution of slavery was defended and justified. To labour meant to enslaved by necessity, and this enslavement was inherent in the conditions of human life. Because men were dominated by the necessities of life, they could win their freedom only through the domination of those whom they subjected to necessity by force. The slave's degradation was a blow of fate and a fate worse than death, because it carried with it a metamorphosis of man into something akin to a tame animal (Arendt, 1998: 83-84).

En su concepción de la necesidad como fuerza natural y brutal que reduce el hombre a su fisicidad y, en consecuencia, a su animalidad, Arendt parece tener en mente las reflexiones del Weil sobre el tema (cf. Fuster, 2013: 41-42). Sin embargo, en este punto los caminos de las dos autoras divergen de forma sustancial. A través de la distinción entre animal laborans (el hombre que se dedica al trabajo), homo faber (el hombre productor) y animal rationale (el hombre que se dedica a la actividad verdaderamente humana de la política), Arendt pretende separar el ámbito de lo político -concebido como aquella esfera en la que, a través de la acción, se ejerce la libertad- del ámbito de lo social, en el que se ejercen las actividades productivas y reproductivas relacionadas con la necesidad.

1 En la utilización del pensamiento de Arendt como herramienta crítica para analizar el de Weil, seguimos la sugerencia que Roberto Esposito expone en el libro que dedica a las dos autoras: «L'una pensa nel rovescio dell'altra: nell'ombra della sua luce, nel silenzio della sua voce, nel vuoto del suo pieno. Pensa ciò che il pensiero dell'altra esclude non come ciò che le è estraneo, ma piuttosto come ciò che appare impensabile, e perciò stesso resta da pensare»; véase: Esposito $(1996,10)$. Se han dedicado varios ensayos a la comparación entre el pensamiento de las dos pensadoras; entre ellos recordamos: Gabellieri (1999), Cedronio (1996), Chenavier (1989).

2 La argumentación se basa sobre la idea de que la representación sea la categoría central de la política moderna o, dicho de otra manera, que la política moderna coincida con su representación. En este sentido, el papel que ocupa la corporeidad en el pensamiento de Weil en tanto crítica del aparato conceptual moderno puede entenderse como crítica, sublimada en la elección de una figura del mundo clásico, de su representación. Sobre el tema de la representación, véase: Galli (1987). 
La pensadora propone de tal manera una representación de la libertad como característica de la condición humana en cuanto distinta de una necesidad que vincula el hombre a su condición animal.

Dejando, por ahora, de lado el análisis arendtiano -sobre el que mucho se habría que añadir- lo que resulta interesante destacar es el hecho de que el trabajo (le travail) en la concepción de Weil reúne tanto las actividades del animal laborans, que tienen como objeto la reproducción de la vida biológica, cuanto aquellas del homo faber, dirigidas a la creación y a la fabricación de objetos cuyo destino va más allá de la urgencia metabólica del consumo. No obstante, el trabajo, para Weil, no se reduce exclusivamente a eso: instrumento de un autentico contacto con el mundo, representa también la actividad que acoge el potencial transformador de la acción política. En el trabajo obrero, trabajo y obra coinciden y, en su coincidencia, posibilitan la acción en cuanto actividad propiamente humana y política. Weil recupera (marxianamente) la productividad política de lo social, mientras que Arendt vuelve justamente al mundo clásico para desechar esa posibilidad y, de esa manera, tratar de superar la concepción moderna de la política. Desde el punto de vista arendtiano, Weil reproduce un defecto típico de la tradición filosófica occidental que niega la autonomía de lo político; sin embargo, esta exclusión es lo que le permite a Weil rescatar políticamente aquel cuerpo dolido, aquel hombre reducido a su animalidad, que el sistema moderno de la gran industria capitalista re-produce sin parar. Por lo tanto, el problema de Weil es el de encontrar la manera a través de la cual la acción política y el ejercicio de la libertad sean posibles a pesar del efecto devastador del trabajo sobre el cuerpo y sobre la mente. La lectura de un pasaje del diario de fábrica que Weil redactó durante su año de trabajo como obrera ayuda a comprender mejor su posicionamiento:

L'épuisement finit par me faire oublier les raisons véritables de mon séjour en usine, rende presque invincible pour moi la tentation la plus forte que comporte cette vie: celle de ne pas plus penser, seul et unique moyen de ne pas souffrir. C'est seulement le samedi après-midi et le dimanche que me reviennent des souvenirs, des lambeaux d'idées, que je me souviens que je suis aussi un être pensant. Effroi qui me saisit en constatant la dépendance où je me trouve à l'égard des circonstances extérieures: il suffirait qu'elles me contraignent un jour à un travail sans repos hebdomadaire - ce qui après tout est toujours possible - et je deviendrais un bête de somme, docile et résignée [...] Je ne suis pas loin de conclure que le salut de l'âme d'un ouvrier dépend d'abord de sa constitution physique (Weil, 1991b : 192-193).

Las observaciones de Weil se parecen a las de Arendt. El trabajo parece haber quedado reducido a la corporeidad, mientras que la corporeidad, subyugada a la necesidad, transforma el trabajador en algo como un animal de carga. Por ello, diría Arendt, los antiguos griegos consideraban la esclavitud como algo más que una condición y creían que implicaba un cambio en la naturaleza del hombre: lo que los hombres compartían con otras formas de vida animal no podía considerarse humano (cf. Arendt, 1998: 80). Weil pone el acento, asimismo, en la deshumanización de lo humano hasta el punto en que se transforma en el problema político principal: 
La révolte est impossible, sauf par éclairs [...] On est seul avec son travail, on ne pourrait se révolter que contre lui - or travailler avec irritation, ce serait mal travailler, donc crever de faim [...] On est comme les chevaux qui se blessent eux même dès qu'ils tirent sur le mors- et on se courbe. On perde même conscience de cette situation, on la subit, c'est tout. Tout réveil de la pensée est alors douloureux (Weil, 1991b: 193).

Weil se pregunta cómo sería posible una rebelión obrera, cuáles las condiciones de libertad de aquellos que viven de tal manera: ¿cómo emancipar a los que son esclavos del sufrimiento del cuerpo, a los que han quedado reducidos a dóciles animales de carga? ¿Cómo puede mejorarse el destino de aquellos que sufren en su propia piel las consecuencias del ejercicio obsceno del poder? La respuesta weiliana es la siguiente: "il faut bien, il me semble, commencer par leur faire relever la tête" (Weil, 2002: 232).

\section{Cuerpo y tragedia}

Puesto que para Weil una revolución comunista no solucionaría el problema de la opresión, su investigación se centra en la posibilidad de modificar el papel de los obreros en el sistema de producción:

La question, pour l'instant, est de savoir si, dans les conditions actuelles, on peut arriver dans le cadre d'une usine à ce que les ouvriers comptent et aient conscience de compter pour quelques chose (Weil, 2002: 223-224).

La primera condición para un trabajo no servil consiste en la estimulación del pensamiento y de la inteligencia, lo que es idéntico a tomar consciencia de la necesidad a la que se está sometidos: "Il me semble que n'importe quelle souffrance est moins accablante, risque moins de dégrader, quand on conçoit le mécanisme des nécessités qui la causent" (Weil, 2002: 224).

Con este espíritu, en 1936, Weil empieza una corta colaboración con Victor Bernard, ingeniero de las oficinas Fonderie de Rosières. La idea de Weil es proponer una serie de reformas a fin de mejorar las condiciones de vida de los trabajadores. En sus propuestas, la filósofa empieza con la idea de que lo que degrada la inteligencia degrada a todo el hombre y que por ello: "le travail doit tendre, dans toute la mesure des possibilités matérielles, à constituer une éducation" (Weil, 2002: 237). Para que esto sea posible es necesario buscar una forma de despertar la inteligencia y la atención de aquellos a los que el trabajo mismo empuja hacia la perdida de consciencia. La colaboración con Bernard, antes de interrumpirse de forma brusca, por la indisposición del ingeniero hacia las ideas políticas demasiado "subversivas" de la filosofa (cf. Weil, 2002: 248-251), permite a Weil desarrollar algunas de sus ideas. Entre ellas, la pensadora trabaja en una serie de artículos a publicarse en la gaceta de fábrica Entre nous en los que presenta la tragedia griega al público de los obreros por medio de la traducción y el comentario de algunos textos y pasajes:

Heureusement il m'est revenue à la mémoire un vieux projet qui me tient vivement à coeur, celui de rendre les chefs-d'oeuvre de la poésie grecque (que j'aime passionné- 
ment) accessibles aux masses populaires. J'ai senti, l'an dernier, que la grande poésie grecque serait cent fois plus proche du peuple, s'il pouvait la connaître, que la littérature française classique et moderne. J'ai commencé par Antigone. Si j'ai réussi dans mon dessein, cela doit pouvoir intéresser et toucher tout le monde -depuis le directeur jusqu'au dernier manoeuvre; et celui-ci doit pouvoir pénétrer là-dedans presque de plain-pied, et cependant sans savoir jamais l'impression d'aucune condescendance, d'aucune effort accompli pour se mettre à sa portée. C'est ainsi que je comprends la vulgarisation. Mais j'ignore si j'ai réussi (Weil, 2002 : 244).

La filosofa comienza a trabajar en tres artículos respectivamente sobre Antígona, Electra y Filoctetes de Sófocles. Finalmente sólo llega a publicarse el artículo sobre Antígona, mientras que el que redacta sobre Electra permanece inédito y el de Filoctetes se queda inacabado. A través de la presentación de historias de sufrimiento y de opresión en los antiguos dramas, Weil intenta, claro está, esquivar la censura de los dirigentes de la oficina: "J'espère cependant que vous n'irez pas jusqu'à trouver Sophocle subversif " (Weil, 2002: 244), le contesta Weil irónicamente al ingeniero Bernard. Sin embargo, remontarse a Sófocles no es solamente un truco determinado por las circunstancias, sino que apunta hacia una calidad intrínseca de la tragedia en general y de los dramas de Sófocles en particular que le permite instruir y educar a la vez que favorece una toma de conciencia por parte de los obreros sobre su propia dramática condición. Así Weil presenta las tragedias:

Dans chacun de ces drames, le personnage principal est un être courageux et fier qui lutte tout seul contre une situation intolérablement douloureuse; il fléchit sous le poids de la solitude, de la misère, de l'humiliation, de l'injustice; par moments son courage se brise; mais il tient bon et ne se laisse jamais dégrader par le malheur. Aussi ces drames, quoique douloureux, ne laissent-ils jamais une impression de tristesse. On en garde plutôt une impression de sérénité (Weil, 1991c: 334).

Según la perspectiva de Weil, la tragedia es capaz de dialogar con las masas obreras porque el drama de la existencia humana se presenta en ella como el enfrentamiento aparente entre libertad y necesidad, un enfrentamiento que, aunque no tenga posible composición mundana, puede reflejar lo que el trabajo encierra como su esencia ${ }^{3}$. La visión trágica que Weil ofrece de la condición obrera, y de la historia con ella, no coincide con una mera aceptación de las condiciones actuales: comprensión y atención, para la filósofa, nunca son sinónimos de resignación, ni tampoco de sumisión. Para Weil, se trata más bien de liberar el potencial de un elemento que no puede seguir siendo políticamente irrelevante. Si el trabajo animaliza el hombre, si el sufrimiento físico embota la inteligencia, la solución no puede ser la de la política moderna, es decir, no puede ser una práctica política orientada hacia el ejercicio de un poder que, por su naturaleza, siempre es cómplice de la opresión. Por medio del lenguaje de la tragedia, Weil busca una forma de comunicar lo que no puede

3 Para una interpretación de las lecturas weilianas de las tragedias relacionada con los temas de la libertad y de la opresión, véase: Fraisse (1993: 23-32). 
ser comunicado a través del lenguaje político corriente, vinculado con la gramática del poder, e incapaz de expresar realmente el sentido último de la opresión ${ }^{4}$.

En el artículo sobre la Electra, más que en los otros, se hace evidente este intento. La elección de la versión sofoclea del drama está determinada por aquellas características del texto que lo vuelven funcional al objetivo de la filosofa ${ }^{5}$. En efecto, la tragedia de Sófocles presenta significativas diferencias con respecto a la de Esquilo (cf. Konstan, 2008: 77). En Coéforos de Esquilo Orestes mata a Egisto y Clitemnestra obedeciendo a un orden del dios Apolo. La necesidad de seguir el oráculo es lo que empuja el protagonista hacia el homicidio, a pesar del horror que inspira el matricidio. Por el contrario, Sófocles suprime el oráculo y de esta manera libera a sus héroes y los hace responsables por sus acciones. Además, Electra se transforma en la víctima de las humillaciones por parte de su madre. Herida en el orgullo y animada por el deseo de venganza, Electra odia a Clitemnestra y lanza gritos de júbilo a la noticia de la muerte de su madre. La Electra de Sófocles es una figura compleja, en la cual la sed de venganza se une con los rasgos de lo inhumano. Sin embargo, Sófocles trata el homicidio como un acto de justicia, determinado por la conciencia.

La libertad y la responsabilidad de los personajes, el énfasis puesta en el sufrimiento físico, el tema de la conciencia de la propia condición y de la justicia son las características de la obra que atraen la atención de Weil. Electra dolida, herida, humillada, envejecida por la desgracias y las penas sufridas, es la heroína que la filósofa necesita para representar el destino del obrero, el esclavo moderno. Este contratiempo, este anacronismo interrumpe el recurrido lineal de la historia: la atención hacia el cuerpo que sufre traslada el punto de vista desde las condiciones materiales de la opresión hacia la desgracia de tal manera que la fuerza que oprime llega a presentarse como algo universal, como la otra cara de la necesidad natural. De tal manera, Weil puede presentar a Electra como a una hermana de aquellos que sufren las injusticias a diario en las oficinas:

Cette histoire d'Électre est bien fait pour toucher tous ces qui, au cours de leur vie, ont eu l'occasion de savoir ce que c'est que d'être malheureux. Bien sûr, cette histoire est une très veille histoire. Mais la misère, et l'humiliation, et l'injustice, et le sentiment qu'on est tout seul, qu'on est livré au malheur, abandonné de Dieu et des hommes, ces choses-là ne sont pas vieilles. Elles son de tout le temps. Ce sont de choses que la vie inflige tous les jours à ceux qui n'ont pas de chance (Weil, 1991a: 339-340).

4 Véase, Revilla (2003: 153): "La 'enseñanza' de los griegos no sólo le va a proporcionar una experiencia que, plenamente asumida, es determinante respecto a problemas concretos; le proporciona también un 'lenguaje' que hace propio. En realidad, el lenguaje weiliano es suyo: habla desde sí mismo, y desde sí busca reordenar el mundo, tarea en la que las metáforas y los símbolos desempeñaran una función especifica". Sobre la corrosión del lenguaje moderno de la política, véase: Esposito (1996: 70).

5 Cabe destacar que Weil, a lo largo de los años, no deja de leer las mismas tragedias volviendo a incorporar en ellas nuevos significados a medida de que su reflexión va cambiando y orientándose hacia contenidos espirituales y religiosos. Véase: Weil (2009: 156-161). 
Con su lectura, Weil enfoca una de las característica principales del drama de Sófocles: la importancia en la puesta en escena del cuerpo de Electra, verdadero protagonista de la tragedia. El cuerpo de Electra -cuya vulnerabilidad lo transforma en símbolo de todos aquellos cuerpos que no pueden ser políticamente correctos y que, por lo tanto, disturban e inquietan los mecanismos simbólicos del poder (cf. Forti, 2015)- representa un cuerpo deshumanizado, objetivado en su reducción a encarnación somática del sufrimiento (cf. Montgomery Griffiths, 2011). El cuerpo de Electra es la sublimación perfecta de aquella fuerza que reduce los hombres a cosas y que Weil no deja de invocar como el origen de toda perversión de la justicia y, por lo tanto, resulta particularmente apto para representar una situación como aquella de la oficina en la que: "Les choses jouent le rôle des hommes, les hommes jouent le rôle de choses; c'est la racine du mal" (Weil, 2002: 295).

La imagen del cuerpo de Electra tiene tanta importancia en el drama que se ha llegado a afirmar que: "Electra is a play of performative trauma, inextricably linked to the "meaningfulness' of the body as a contested site of power and control" (Montgomery Griffiths, 2011: 231). El cuerpo opera en la tragedia de manera performativa, deslizándose entre subjetividad y objetividad. Por su medio, Electra construye su propia identidad: usando y abusando de las huellas dejadas por la desgracia y el sufrimiento. Las privaciones a las que está sometida, así pues, no representan solamente signos de pasividad, sino que se muestran también como símbolos de una silenciosa lucha, en la que el ayuno y la indigencia se transforman en sinónimos de resistencia, rigor moral, autonomía e integridad. De hecho, es lo que de inhumano hay en su condición lo que empuja Electra a la acción. De esta manera, Weil está autorizada a darle relevancia a la representación de una heroína desfigurada por las privaciones: el cuerpo deshecho muestra las plagas del hambre, de la malnutrición; el sufrimiento no encuentra consolación, ni solidaridad; la belleza se ha ido para siempre:

Déjà la meilleure part de ma vie est passée, écoulée dans le désespoir. Je n'en puis plus.

Privée de parents, le chagrin me ronge.

Il n'y a pas d'homme qui m'aime et me protege.

Il me faut, comme la dernière des servants,

Travailler dans la maison de mon père;

Habillée de ces haillons humiliants

je dois rester debout autor de tables vide ${ }^{6}$

El cuerpo de Electra, cuerpo sexuado, cuerpo femenino le parece a Weil -por otro lado indiferente en relación a la cuestión feminista, aunque consciente de la mayor opresión sufrida por las mujeres- especialmente adecuado para representar la violencia obscena de un poder que, en su capacidad de reducir aquellos que lo sufren a una condición subhumana, manifiesta su vínculo esencial con la violencia. Un vínculo que, en el pensamiento de Weil, transforma el poder en una fuerza universal. Una fuerza que no tiene historia, que atraviesa las épocas en cuanto ley del universo material y que la política renovada, entendida como

6 La traducción es la de Weil (1991a: 342). 
búsqueda de la justicia y no como ejercicio del poder, puede llegar a romper: la justicia es real porque está presente en el corazón de los hombres (cf. Weil, 2013: 347). Electra conserva su deseo de libertad y justicia, a pesar de todo. En tal modo se dirige Electra a su hermana que, por el contrario, ha tomado la decisión de someterse, apagando el corazón y la inteligencia:
Non, non, jamais, en aucun cas, quand on devrait
m'accorder ces faveurs dont te voilà si fière
je ne céderai à ces gens-là. À toi la vie abondante.
Ne pas faire violence à mon coeur, ce sera là
ma nourriture. Je n'envie pas tes privilèges (Weil, 1991a :343).

Este pasaje permite una lectura política de la feminidad resistente de Electra, por contraste con la imagen sumisa de Crisótemis, portadora de una feminidad en línea con los estándares del tiempo (cf. Wheeler, 2003: 279) y permite también una lectura política de la tragedia en su conjunto, poniendo de relieve aquellos elementos que pueden favorecer su interpretación en términos de una alegoría de la situación política de la Atenas de la época: por ejemplo, la presencia, poco usual en la tragedia del sustantivo eleutheria para indicar la libertad en su sentido propiamente político de libertad desde la tiranía (cf. Konstan, 2008: 79). Weil capta todos estos elementos y los reúne. Su Electra se transforma en un himno a la resistencia: el héroe es aquello que, aunque esté a punto de doblarse, vuelve a encontrarse en el medio del sufrimiento. Al final de la tragedia: "L'oppression est enfin brisée. Électre est libre" (Weil, 1991a: 348). Este es el desafío que el pensamiento weiliano no dejará de ponerse a sí mismo en los años siguientes.

\section{Bibliografía}

Arendt,Hannah (1998): The Human Condition, University of Chicago Press, Chicago\&London. Bottici, Chiara (2015): «Rethinking the Biopolitical Turn. From the Thanatopolitical to the Geneapolitical Paradigm», Graduate Philosophy Journal (Nueva York), nº 1, pp. 175-198.

Galli, Carlo (1987): «Immagine e rappresentanza politica», Filosofia politica (Bolonia), ${ }^{\circ}$ 1, pp. 9-29.

Cedronio, Marina (1996): Modernité, démocratie et totalitarisme: Simone Weil et Hannah Arendt, Klincksieck, París.

Chenavier, Robert (1989): «Simone Weil e Hannah Arendt», Cahiers Simone Weil (París), $\mathrm{n}^{\mathrm{o}} 2$, pp. 149-169.

Chenavier, Robert (2001): Simone Weil. Une philosophie du travail, Éditions du Cerf, París.

Esposito, Roberto (1996): L'origine della politica. Hannah Arendt o Simone Weil?, Donzelli Editore, Roma.

Forti, Simona (2015): «Democratic bodies. Biopolitically Correct», Graduate Faculty Philosophy Journal, $\mathrm{n}^{\circ} 1$, pp. 145-158.

Fraisse, Simone (1993): «Oppressione e libertà: una lettura weiliana della tragedia greca», en: A. Marchetti (ed.), Politeia e sapienza. In questione con Simone Weil, Pàtron, Bologna, pp. 23-32. 
Fuster, Àngela Lorena (2013): «Sin prejucio ni sentimentalismo. Hannah Arendt, lectora de Simone Weil», en: F. Birulés y R. Rius Gatell (eds.): Lectoras de Simone Weil, Icaria, Barcelona, pp. 19-50.

Gabellieri, Emmanuel (1999): «"Vie publique” et "vita activa” chez Simone Weil et Hannah Arendt», en: Cahiers Simone Weil (París), n 2, pp. 135-152.

Konstan, David (2008): «Sophocles'Electra as Political Allegory: A Suggestion», Classical Philology (Chicago), nº pp. 77-80.

Montgomery Griffiths, Jane (2011): «The Abject Eidos. Trauma and the Body in Sophocles' Electra», en: J. Parker y T. Mathews (eds.), Tradition, Translation, Trauma. The Classic and the Modern, Oxford University Press, Oxford, pp. 229-243.

Revilla, Carmen (2003): Simone Weil: nombrar la experiencia, Trotta, Madrid.

Weil, Simone (1988): «Science et perception dans Descartes», en: Ead.: Oeuvres Complètes, I, 1, Gallimard, París, pp. 161-221.

Weil, Simone (1991a): «Électre», en: Ead.: Oeuvres Complètes, II, 2, Gallimard, París, pp. 339-348.

Weil, Simone (1991b): «Journal d'usine», en: Ead.: Oeuvres Complètes, II, 2, Gallimard, París, pp. 151-310.

Weil, Simone (1991c): «Antigone», en: Ead.: Oeuvres Complètes, II, 2, Gallimard, París, pp. 333-338.

Weil, Simone (2002): La condition ouvrière, Gallimard, París.

Weil, Simone (2009): «Intuitions pré-chrétiennes», en: Ead., Oeuvres Complètes, IV, 2, Gallimard, París, pp. 156-161.

Weil, Simone (2013): «L'Enracinement. Prélude à un déclaration des devoirs envers l'être humain» en: Ead.: Oeuvres Complètes, V, 2, Gallimard, París.

Wheeler, Graham (2003): «Gender and Transgression in Sophocles' Electra», The Classical Quarterly (Cambridge), n 2, pp. 377-388. 